\title{
Brane-bulk energy exchange : a model with the present universe as a global attractor
}

\author{
Georgios Kofinas $^{1 *}$, Grigorios Panotopoulos ${ }^{2 \dagger}$ and Theodore N. Tomaras ${ }^{2,3 \ddagger}$ \\ ${ }^{1}$ Departament de Física Fonamental, Universitat de Barcelona, Diagonal 647, 08028 Barcelona, Spain \\ ${ }^{2}$ Department of Physics and Institute of Plasma Physics, \\ University of Crete, 71003 Heraklion, Greece and \\ ${ }^{3}$ Foundation of Research and Technology, Hellas, 71110 Heraklion, Greece
}

(Dated: September 29, 2018)

\begin{abstract}
The role of brane-bulk energy exchange and of an induced gravity term on a single braneworld of negative tension and vanishing effective cosmological constant is studied. It is shown that for the physically interesting cases of dust and radiation a unique global attractor which can realize our present universe (accelerating and $0<\Omega_{m 0}<1$ ) exists for a wide range of the parameters of the model. For $\Omega_{m 0}=0.3$, independently of the other parameters, the model predicts that the equation of state for the dark energy today is $w_{D E, 0}=-1.4$, while $\Omega_{m 0}=0.03$ leads to $w_{D E, 0}=-1.03$. In addition, during its evolution, $w_{D E}$ crosses the $w_{D E}=-1$ line to smaller values.
\end{abstract}

\section{INTRODUCTION}

In cosmologies where the present universe is realized as a finite point during the cosmic evolution, the answer to the coincidence question "why it is that today $\Omega_{m 0}$ and $\Omega_{D E, 0}$ are of the same order of magnitude", relies on appropriate choice of initial conditions. By contrast, in a scenario in which the present universe is in its asymptotic era (close to a fixed point) the answer to the above question reduces to an appropriate choice of the parameters of the model. However, this latter situation is not easily realized if today's universe is accelerating, because:

If the energy density of a perfect fluid with equation of state $w>-1 / 3$ of any cosmological system is conserved, all fixed points of the system with $\Omega_{m} \neq 0$ are decelerating.

Indeed, with $\rho$ the energy density of the perfect fluid with conservation equation $\dot{\rho}+3(1+w) H \rho=0$, the Hubble equation of an arbitrary cosmology can be written in the form

$$
H^{2}=2 \gamma\left(\rho+\rho_{D E}\right),
$$

where $\gamma=4 \pi G_{N} / 3$. Then, the equation governing $\rho_{D E}$ can always be brought into the form $\dot{\rho}_{D E}+3(1+$ $\left.w_{D E}\right) H \rho_{D E}=0$, where $w_{D E}$ is time-dependent and distiguishes one model from the other. It can be easily seen that $d\left(\Omega_{m} / \Omega_{D E}\right) / d \ln a=3\left(\Omega_{m} / \Omega_{D E}\right)\left(w_{D E}-w\right)$ and $2 q=1+3\left(w \Omega_{m}+w_{D E} \Omega_{D E}\right)$, where $\Omega_{m}=2 \gamma \rho / H^{2}$, $\Omega_{D E}=2 \gamma \rho_{D E} / H^{2}$ and $q=-\ddot{a} / a H^{2}$. At the fixed point (denoted by $*$ ) $d\left(\Omega_{m} / \Omega_{D E}\right) / d \ln a=0$. For $\Omega_{m *} \neq 0$ one obtains $w_{D E *}=w$, and $2 q_{*}=1+3 w>0$.

Thus, independently of the cosmological model, the only way our accelerating universe with $\Omega_{m *} \neq 0$ can be close to a late time fixed point is by violating the standard

\footnotetext{
*kofinas@ffn.ub.es

†panotop@physics.uoc.gr

†tomaras@physics.uoc.gr
}

conservation equation of matter. In 4-dimensional theories, an accelerating late time cosmological phase characterized by a frozen ratio of dark matter/dark energy appears in coupled dark energy scenarios [1] as a result of the interaction of the dark matter with other energymomentum components, such as scalar fields. In higher dimensional theories, where the universe is represented as a 3-brane, this violation could be the result of energy exchange between the brane and the bulk. In particular in five dimensions, a universe with fixed points characterized by $\Omega_{m *} \neq 0, q_{*}<0$ was realized in [2] in the context of the Randall-Sundrum braneworld scenario with energy influx from the bulk. However, these fixed points cannot represent the present universe, since they have $\Omega_{m *}>2$. In this paper we present a brane-bulk energy exchange model with induced gravity whose global attractor can represent today's universe.

Let us consider an arbitrary cosmology in the form (1). Instances of such cosmologies arise in braneworld models or in theories with modified 4-dimensional actions leading to $H^{2}=f(\rho)$, or in cosmologies where $\rho_{D E}$ is due to additional fields. Assuming that as a result of some interaction $\rho$ is not conserved, it will satisfy an equation of the form

$$
\dot{\rho}+3(1+w) H \rho=-T .
$$

Then, the equation governing $\rho_{D E}$ can always be brought into the form

$$
\dot{\rho}_{D E}+3\left(1+w_{D E}\right) H \rho_{D E}=T,
$$

where $w_{D E}$ is time and model dependent. Whenever a fixed point of the system satisfies

$$
H_{*} T_{*} \neq 0 \quad, \quad \dot{\rho}=\dot{\rho}_{D E}=0,
$$

one obtains

$$
w_{D E *}=-1-\frac{1+w}{\Omega_{m *}^{-1}-1} .
$$

Equation (5) is model-independent, in the sense that it does not depend on the form of $T$ or the function $w_{D E}(t)$. 
For $\Omega_{m *}<1$ equation (5) gives $w_{D E *}<-1$. Specifically, for $w=0$ and $\Omega_{m *}=\Omega_{C D M}=0.3$ one obtains $w_{D E *}=$ -1.4 , while for $\Omega_{m *}=\Omega_{b a r}=0.03, w_{D E *}=-1.03$.

The cosmology discussed in the present paper has a global attractor of the form (44), (5] [3]. Moreover, the universe during its evolution crosses the $w_{D E}=-1$ barrier from higher values. This behavior is favored by several recent model-independent [4] as well as modeldependent [5, 6, 7, 8] analyses of the astronomical data.

\section{THE MODEL}

We consider the model described by the gravitational brane-bulk action [9]

$$
S=\int d^{5} x \sqrt{-g}\left(M^{3} R-\Lambda\right)+\int d^{4} x \sqrt{-h}\left(m^{2} \hat{R}-V\right),
$$

where $R, \hat{R}$ are the Ricci scalars of the bulk metric $g_{A B}$ and the induced metric $h_{A B}=g_{A B}-n_{A} n_{B}$ respectively $\left(n^{A}\right.$ is the unit vector normal to the brane and $A, B=0,1,2,3,5)$. The bulk cosmological constant is $\Lambda / 2 M^{3}<0$, the brane tension is $V$, and the inducedgravity crossover scale is $r_{c}=m^{2} / M^{3}$.

We assume the cosmological bulk ansatz

$$
d s^{2}=-n(t, y)^{2} d t^{2}+a(t, y)^{2} \gamma_{i j} d x^{i} d x^{j}+b(t, y)^{2} d y^{2},
$$

where $\gamma_{i j}$ is a maximally symmetric 3 -dimensional metric, parametrized by the spatial curvature $k=-1,0,1$. The non-zero components of the five-dimensional Einstein tensor are

$$
\begin{aligned}
G_{00} & =3\left\{\frac{\dot{a}}{a}\left(\frac{\dot{a}}{a}+\frac{\dot{b}}{b}\right)-\frac{n^{2}}{b^{2}}\left[\frac{a^{\prime \prime}}{a}+\frac{a^{\prime}}{a}\left(\frac{a^{\prime}}{a}-\frac{b^{\prime}}{b}\right)\right]+\frac{k n^{2}}{a^{2}}\right\}(8) \\
G_{i j} & =\frac{a^{2}}{b^{2}} \gamma_{i j}\left\{\frac{a^{\prime}}{a}\left(\frac{a^{\prime}}{a}+\frac{2 n^{\prime}}{n}\right)-\frac{b^{\prime}}{b}\left(\frac{n^{\prime}}{n}+\frac{2 a^{\prime}}{a}\right)+\frac{2 a^{\prime \prime}}{a}+\frac{n^{\prime \prime}}{n}\right\} \\
+ & \frac{a^{2}}{n^{2}} \gamma_{i j}\left\{\frac{\dot{a}}{a}\left(\frac{2 \dot{n}}{n}-\frac{\dot{a}}{a}\right)-\frac{2 \ddot{a}}{a}+\frac{\dot{b}}{b}\left(\frac{\dot{n}}{n}-\frac{2 \dot{a}}{a}\right)-\frac{\ddot{b}}{b}\right\}-k \gamma_{i j}(9) \\
G_{05} & =3\left(\frac{n^{\prime}}{n} \frac{\dot{a}}{a}+\frac{a^{\prime}}{a} \frac{\dot{b}}{b}-\frac{\dot{a}^{\prime}}{a}\right) \\
G_{55} & =3\left\{\frac{a^{\prime}}{a}\left(\frac{a^{\prime}}{a}+\frac{n^{\prime}}{n}\right)-\frac{b^{2}}{n^{2}}\left[\frac{\ddot{a}}{a}+\frac{\dot{a}}{a}\left(\frac{\dot{a}}{a}-\frac{\dot{n}}{n}\right)\right]-\frac{k b^{2}}{a^{2}}\right\},(11)
\end{aligned}
$$

where primes indicate derivatives with respect to $y$, while dots derivatives with respect to $t$. The five-dimensional Einstein equations take the usual form

$$
G_{A C}=\left.\frac{1}{2 M^{3}} T_{A C}\right|_{t o t}
$$

where

$$
\left.T_{C}^{A}\right|_{t o t}=\left.T_{C}^{A}\right|_{v, B}+\left.T_{C}^{A}\right|_{m, B}+\left.T_{C}^{A}\right|_{v, b}+\left.T_{C}^{A}\right|_{m, b}+\left.T_{C}^{A}\right|_{i n d}
$$

is the total energy-momentum tensor,

$$
\begin{aligned}
& \left.T_{C}^{A}\right|_{v, B}=\operatorname{diag}(-\Lambda,-\Lambda,-\Lambda,-\Lambda,-\Lambda) \\
& \left.T_{C}^{A}\right|_{v, b}=\operatorname{diag}(-V,-V,-V,-V, 0) \frac{\delta(y)}{b} \\
& \left.T_{C}^{A}\right|_{m, b}=\operatorname{diag}(-\rho, p, p, p, 0) \frac{\delta(y)}{b} .
\end{aligned}
$$

$\left.T_{C}^{A}\right|_{m, B}$ is any possible additional energy-momentum in the bulk, the brane matter content $\left.T_{C}^{A}\right|_{m, b}$ consists of a perfect fluid with energy density $\rho$ and pressure $p$, while the contributions arising from the scalar curvature of the brane are given by

$$
\begin{aligned}
& \left.T_{0}^{0}\right|_{\text {ind }}=\frac{6 m^{2}}{n^{2}}\left(\frac{\dot{a}^{2}}{a^{2}}+\frac{k n^{2}}{a^{2}}\right) \frac{\delta(y)}{b} \\
& \left.T_{j}^{i}\right|_{\text {ind }}=\frac{2 m^{2}}{n^{2}}\left(\frac{\dot{a}^{2}}{a^{2}}-\frac{2 \dot{a} \dot{n}}{a n}+\frac{2 \ddot{a}}{a}+\frac{k n^{2}}{a^{2}}\right) \delta_{j}^{i} \frac{\delta(y)}{b} .
\end{aligned}
$$

Assuming a $\mathbb{Z}_{2}$ symmetry around the brane, the singular part of equations (12) gives the matching conditions

$$
\begin{gathered}
\frac{a_{o^{+}}^{\prime}}{a_{o} b_{o}}=-\frac{\rho+V}{12 M^{3}}+\frac{r_{c}}{2 n_{o}^{2}}\left(\frac{\dot{a}_{o}^{2}}{a_{o}^{2}}+\frac{k n_{o}^{2}}{a_{o}^{2}}\right) \\
\frac{n_{o^{+}}^{\prime}}{n_{o} b_{o}}=\frac{2 \rho+3 p-V}{12 M^{3}}+\frac{r_{c}}{2 n_{o}^{2}}\left(\frac{2 \ddot{a}_{o}}{a_{o}}-\frac{\dot{a}_{o}^{2}}{a_{o}^{2}}-\frac{2 \dot{a}_{o} \dot{n}_{o}}{a_{o} n_{o}}-\frac{k n_{o}^{2}}{a_{o}^{2}}\right)
\end{gathered}
$$

(the subscript o denotes the value on the brane), while from the 05,55 components of equations (12) we obtain

$$
\frac{n_{o}^{\prime}}{n_{o}} \frac{\dot{a}_{o}}{a_{o}}+\frac{a_{o}^{\prime}}{a_{o}} \frac{\dot{b}_{o}}{b_{o}}-\frac{\dot{a}_{o}^{\prime}}{a_{o}}=\frac{T_{05}}{6 M^{3}}
$$

$\frac{a_{o}^{\prime}}{a_{o}}\left(\frac{a_{o}^{\prime}}{a_{o}}+\frac{n_{o}^{\prime}}{n_{o}}\right)-\frac{b_{o}^{2}}{n_{o}^{2}}\left[\frac{\ddot{a}_{o}}{a_{o}}+\frac{\dot{a}_{o}}{a_{o}}\left(\frac{\dot{a}_{o}}{a_{o}}-\frac{\dot{n}_{o}}{n_{o}}\right)\right]-\frac{k b_{o}^{2}}{a_{o}^{2}}=\frac{T_{55}-\Lambda b_{o}^{2}}{6 M^{3}}$,

where $T_{05}, T_{55}$ are the 05 and 55 components of $\left.T_{A C}\right|_{m, B}$ evaluated on the brane. Substituting the expressions (19), (20) in equations (21), (22), we obtain the semiconservation law and the Raychaudhuri equation

$$
\begin{gathered}
\dot{\rho}+3 \frac{\dot{a}_{o}}{a_{o}}(\rho+p)=-\frac{2 n_{o}^{2}}{b_{o}} T_{5}^{0} \\
\left(H_{o}^{2}+\frac{k}{a_{o}^{2}}\right)\left[1-\frac{r_{c}^{2}(\rho+3 p-2 V)}{24 m^{2}}\right]+\frac{r_{c}^{2}(\rho+3 p-2 V)(\rho+V)}{144 m^{4}} \\
+\left(\frac{\dot{H}_{o}}{n_{o}}+H_{o}^{2}\right)\left[1-\frac{r_{c}^{2}}{2}\left(H_{o}^{2}+\frac{k}{a_{o}^{2}}\right)+\frac{r_{c}^{2}(\rho+V)}{12 m^{2}}\right]=\frac{\Lambda-T_{5}^{5}}{6 M^{3}},(24)
\end{gathered}
$$

where $H_{o}=\dot{a}_{o} / a_{o} n_{o}$ is the Hubble parameter of the brane. One can easily check that in the limit $m \rightarrow 0$, equation (24) reduces to the corresponding second order equation of the model without $\hat{R}$ 2]. Energy exchange between the brane and the bulk has also been investigated in [10, 11, 12].

Since only the 55 component of $\left.T_{A C}\right|_{m, B}$ enters equation (24), one can derive a cosmological system that is largely independent of the bulk dynamics, if at the position of the brane the contribution of this component relative to the bulk vacuum energy is much less important than the brane matter relative to the brane vacuum energy, or schematically

$$
\left|\frac{T_{5}^{5}}{\Lambda}\right| \ll\left|\frac{\rho}{V}\right| .
$$


Then, for $|\Lambda|$ not much larger than the Randall-Sundrum value $V^{2} / 12 M^{3}$, the term $T_{5}^{5}$ in equation (24) can be ignored. Alternatively, the term $T_{5}^{5}$ can be ignored in equation (24) if simply

$$
\left|\frac{T_{5}^{5}}{\Lambda}\right| \ll 1 .
$$

Note that relations (25) and (26) are only boundary conditions for $T_{5}^{5}$, which in a realistic description in terms of bulk fields will be translated into boundary conditions on these fields. In the special case where (25), (26) are valid throughout the bulk, the latter remains unperturbed by the exchange of energy with the brane. is

One can now check that a first integral of equation (24)

$$
\begin{aligned}
H_{o}^{4}-\frac{2 H_{o}^{2}}{3}\left(\frac{\rho+V}{2 m^{2}}+\right. & \left.\frac{6}{r_{c}^{2}}-\frac{3 k}{a_{o}^{2}}\right)+\left(\frac{\rho+V}{6 m^{2}}-\frac{k}{a_{o}^{2}}\right)^{2}+ \\
& +\frac{4}{r_{c}^{2}}\left(\frac{\Lambda}{12 M^{3}}-\frac{k}{a_{o}^{2}}\right)-\frac{\chi}{3 r_{c}^{2}}=0,
\end{aligned}
$$

with $\chi$ satisfying

$$
\dot{\chi}+4 n_{o} H_{o} \chi=\frac{r_{c}^{2} n_{o}^{2} T}{m^{2} b_{o}}\left(H_{o}^{2}-\frac{\rho+V}{6 m^{2}}+\frac{k}{a_{o}^{2}}\right),
$$

and $T=2 T_{5}^{0}$ is the discontinuity across the brane of the 05 component of the bulk energy-momentum tensor. The solution of (27) for $H_{o}$ is

$$
H_{o}^{2}=\frac{\rho+V}{6 m^{2}}+\frac{2}{r_{c}^{2}}-\frac{k}{a_{o}^{2}} \pm \frac{1}{\sqrt{3} r_{c}}\left[\frac{2(\rho+V)}{m^{2}}+\frac{12}{r_{c}^{2}}-\frac{\Lambda}{M^{3}}+\chi\right]^{\frac{1}{2}},
$$

and equation (28) becomes

$\dot{\chi}+4 n_{o} H_{o} \chi=\frac{2 n_{o}^{2} T}{m^{2} b_{o}}\left\{1 \pm \frac{r_{c}}{2 \sqrt{3}}\left[\frac{2(\rho+V)}{m^{2}}+\frac{12}{r_{c}^{2}}-\frac{\Lambda}{M^{3}}+\chi\right]^{\frac{1}{2}}\right\}$.

At this point we find it convenient to employ a coordinate frame in which $b_{o}=n_{o}=1$ in the above equations. This can be achieved by using Gauss normal coordinates with $b(t, z)=1$, and by going to the temporal gauge on the brane with $n_{o}=1$. It is also convenient to define the parameters

$$
\begin{aligned}
\lambda & =\frac{2 V}{m^{2}}+\frac{12}{r_{c}^{2}}-\frac{\Lambda}{M^{3}} \\
\mu & =\frac{V}{6 m^{2}}+\frac{2}{r_{c}^{2}} \\
\gamma & =\frac{1}{12 m^{2}} \\
\beta & =\frac{1}{\sqrt{3} r_{c}} .
\end{aligned}
$$

For a perfect fluid on the brane with equation of state $p=w \rho$ our system is described by equations (23), (29),
(30), which simplify to (we omit the subscript o in the following)

$$
\begin{gathered}
\dot{\rho}+3(1+w) H \rho=-T \\
H^{2}=\mu+2 \gamma \rho \pm \beta \sqrt{\lambda+24 \gamma \rho+\chi}-\frac{k}{a^{2}} \\
\dot{\chi}+4 H \chi=24 \gamma T\left(1 \pm \frac{1}{6 \beta} \sqrt{\lambda+24 \gamma \rho+\chi}\right)
\end{gathered}
$$

while the second order equation (24) for the scale factor becomes

$$
\frac{\ddot{a}}{a}=\mu-(1+3 w) \gamma \rho \pm \beta \frac{\lambda+6(1-3 w) \gamma \rho}{\sqrt{\lambda+24 \gamma \rho+\chi}} .
$$

Finally, setting $\psi \equiv \sqrt{\lambda+24 \gamma \rho+\chi}$, equations (36), (37), (38) take the form

$$
\begin{gathered}
H^{2}=\mu+2 \gamma \rho \pm \beta \psi-\frac{k}{a^{2}} \\
\dot{\psi}+2 H\left(\psi-\frac{\lambda+6(1-3 w) \gamma \rho}{\psi}\right)= \pm \frac{2 \gamma T}{\beta} \\
\frac{\ddot{a}}{a}=\mu-(1+3 w) \gamma \rho \pm \beta \frac{\lambda+6(1-3 w) \gamma \rho}{\psi} .
\end{gathered}
$$

Throughout, we will assume $T(\rho)=A \rho^{\nu}$, with $\nu>0, A$ constant parameters [2, 13]. Notice that the system of equations (35)-(37) has the influx-outflow symmetry $T \rightarrow$ $-T, H \rightarrow-H, t \rightarrow-t$. For $T=0$ the system reduces to the cosmology studied in [14].

We will be referring to the upper (lower) \pm solution as Branch A (Branch B). We shall be interested in a model that reduces to the Randall-Sundrum vacuum in the absence of matter, i.e. it has vanishing effective cosmological constant. This is achieved for $\mu=\mp \beta \sqrt{\lambda}$, which, given that $m^{2} V+12 M^{6}$ is negative (positive) for branches A (B), is equivalent to the fine-tuning $\Lambda=-V^{2} / 12 M^{3}$. Notice that for Branch A, $V$ is necessarily negative. Cosmologies with negative brane tension in the induced gravity scenario have also been discussed in [15].

Consider the case $k=0$. The system possesses the obvious fixed point $\left(\rho_{*}, H_{*}, \psi_{*}\right)=(0,0, \sqrt{\lambda})$. However, for $\operatorname{sgn}(H) T<0$ there are non-trivial fixed points, which are found by setting $\dot{\rho}=\dot{\psi}=0$ in equations (35), (40). For $w \leq 1 / 3$ these are:

$$
\begin{gathered}
\frac{2 T\left(\rho_{*}\right)^{2}}{9(1+w)^{2} \rho_{*}^{2}}=2 \mu+(1-3 w) \gamma \rho_{*} \\
\pm \sqrt{9(1+w)^{2} \gamma^{2} \rho_{*}^{2}+4 \beta^{2}\left[\lambda+6(1-3 w) \gamma \rho_{*}\right]} \\
H_{*}=-\frac{T\left(\rho_{*}\right)}{3(1+w) \rho_{*}} \\
\psi_{*}^{2} \pm \frac{3(1+w)}{\beta} \gamma \rho_{*} \psi_{*}-\left[\lambda+6(1-3 w) \gamma \rho_{*}\right]=0 .
\end{gathered}
$$

Equation (41) gives

$$
\left(\frac{\ddot{a}}{a}\right)_{*}=\frac{T\left(\rho_{*}\right)^{2}}{9(1+w)^{2} \rho_{*}^{2}},
$$


which is positive, and also, it has the same form (as a function of $\rho_{*}$ ) as in the absence of $\hat{R}$. The deceleration parameter is found to have the value

$$
q_{*}=-1
$$

which means $\dot{H}_{*}=0$. Furthermore, at this fixed point we find

$$
\Omega_{m *} \equiv \frac{2 \gamma \rho_{*}}{H_{*}^{2}}=\frac{18(1+w)^{2}}{A^{2}} \gamma \rho_{*}^{3-2 \nu} .
$$

Equation (42), when expressed in terms of $\Omega_{m *}$, has only one root for each branch

$$
\rho_{*}=\frac{\beta}{2 \gamma} \frac{6(1-3 w) \beta \pm \sqrt{\lambda}\left(1-3 w-4 \Omega_{m *}^{-1}\right)}{\left(2 \Omega_{m *}^{-1}+1+3 w\right)\left(\Omega_{m *}^{-1}-1\right)} .
$$

However, it can be seen from (48) that for $-1 \leq w \leq 1 / 3$ and $\Omega_{m *}<1$ the Branch B is inconsistent with equation (42). On the contrary, Branch A with $-1 \leq w \leq 1 / 3$ and $\Omega_{m *}<1$ is consistent for $0<6(1-3 w) \beta+\sqrt{\lambda}(1-$ $\left.3 w-4 \Omega_{m *}^{-1}\right)<3 \sqrt{4(1-3 w)^{2} \beta^{2}-(1+w)^{2} \lambda}$. Thus, since we are interested in realizing the present universe as a fixed point, Branch B should be rejected, and from now on we will only consider Branch A. So, we have seen until now that for negative brane tension, we can have a fixed point of our model with acceleration and $0<\Omega_{m *}<1$. This behavior is qualitatively different from the one obtained in the context of the model presented in 2] (for $-1 / 3 \leq w \leq 1 / 3)$, where for positive brane tension we have $\Omega_{m *}>2$, while for negative brane tension the universe necessarily exhibited deceleration; therefore, in that model the idea that the present universe is close to a fixed point could not be realized.

Concerning the negative brane tension the following remarks are in order: (a) In the conventional, nonsupersymmetric setting, it is well known that a negative tension brane with or without induced gravity is accompanied by tachyonic bulk gravitational modes [16]; however, including the Gauss-Bonnet corrections relevant at high-energies, the tachyonic modes can be completely removed for a suitable range of the parameters [17]. (b) As shown in [18], in supersymmetric theories, spacetimes with two branes of opposite tension are stable; in particular, there is no instability due to expanding "balooning" modes on the negative brane. It is, however, unclear what happens in models with supersymmetry unbroken in the bulk but softly broken on the brane. (c) Finally, it has been shown [19] that with appropriate choice of boundary conditions, both at the linearized level as well as in the full theory, the gravitational potential of a mass on a negative tension brane has the correct $1 / r$ attractive behaviour.

\section{CRITICAL POINT ANALYSIS}

We shall restrict ourselves to the flat case $k=0$. In order to study the dynamics of the system, it is convenient to use (dimensionless) flatness parameters such that the state space is compact [20]. Defining

$$
\omega_{m}=\frac{2 \gamma \rho}{D^{2}} \quad, \quad \omega_{\psi}=\frac{\beta \psi}{D^{2}} \quad, \quad Z=\frac{H}{D}
$$

where $D=\sqrt{H^{2}-\mu}$, we obtain the equations

$$
\begin{gathered}
\omega_{m}+\omega_{\psi}=1 \\
\omega_{m}^{\prime}=\omega_{m}\left[(1+3 w)\left(\omega_{m}-1\right) Z-\frac{A}{\sqrt{|\mu|}}\left(\frac{|\mu| \omega_{m}}{2 \gamma}\right)^{\nu-1}\left(1-Z^{2}\right)^{\frac{3}{2}-\nu}\right. \\
\left.-2 Z\left(1-Z^{2}\right) \frac{1-Z^{2}-3(1-3 w) \beta^{2} \mu^{-1} \omega_{m}}{1-\omega_{m}}\right](51) \\
Z^{\prime}=\left(1-Z^{2}\right)\left[\left(1-Z^{2}\right) \frac{1-Z^{2}-3(1-3 w) \beta^{2} \mu^{-1} \omega_{m}}{1-\omega_{m}}-1\right. \\
\left.-\frac{1+3 w}{2} \omega_{m}\right],(52)
\end{gathered}
$$

with $^{\prime}=d / d \tau=D^{-1} d / d t$. Note that $-1 \leq Z \leq 1$, while both $\omega$ 's satisfy $0 \leq \omega \leq 1$. The deceleration parameter is given by

$q=\frac{1}{Z^{2}}\left[\frac{1+3 w}{2} \omega_{m}-\left(1-Z^{2}\right) \frac{\omega_{m}-Z^{2}-3(1-3 w) \beta^{2} \mu^{-1} \omega_{m}}{1-\omega_{m}}\right]$

and $H^{\prime}=-H Z(q+1)$. The system of equations (51)-(52) inherits from equations (35)-(37) the symmetry $A \rightarrow-A$, $Z \rightarrow-Z, \tau \rightarrow-\tau$. The system written in the new variables contains only three parameters. However, going back to the physical quantities $H, \rho$ one will need specific values of two more parameters.

It is obvious that the points with $|Z|=1$ have $H=\infty$. Therefore, from (39) it arises that the infinite density $\rho=$ $\infty$ big bang (big crunch) singularity, when it appears, is represented by one of the points with $Z=1(Z=-1)$. The points with $\omega_{m}=1,|Z| \neq 1,0$ have $\omega_{m}^{\prime}=\infty, Z^{\prime}=\infty$ and finite $\rho, H$; for $w \leq 1 / 3$, one has in addition $\ddot{a} / a=+\infty$, i.e. divergent $4 \mathrm{D}$ curvature scalar on the brane.

The system possesses, generically, the fixed point (a) $\left(\omega_{m *}, \omega_{\psi *}, Z_{*}\right)=(0,1,0)$, which corresponds to the fixed point $\left(\rho_{*}, H_{*}, \psi_{*}\right)=(0,0, \sqrt{\lambda})$ discussed above. For $\nu \leq 3 / 2$ there are in addition the fixed points (b) $\left(\omega_{m *}, \omega_{\psi *}, Z_{*}\right)=(0,1,1)$ and (c) $\left(\omega_{m *}, \omega_{\psi *}, Z_{*}\right)=$ $(0,1,-1)$. All these critical points are either nonhyperbolic, or their characteristic matrix is not defined at all; thus, their stability cannot be studied by first order perturbation analysis. In cases like these, one can find non-conventional behaviors (such as saddlenodes and cusps 21]) of the flow-chart near the critical points. There are two more candidate fixed points (d) $\left(\omega_{m *}, \omega_{\psi *}, Z_{*}\right)=(1,0,1)$ and (e) $\left(\omega_{m *}, \omega_{\psi *}, Z_{*}\right)=$ $(1,0,-1)$, whose existence cannot be confirmed directly from the dynamical system, since they make equations (51), (52) undetermined. Apart from the above, there 
are other critical points given by

$$
\begin{gathered}
\frac{A}{\sqrt{|\mu|}}\left(\frac{|\mu| \omega_{m *}}{2 \gamma}\right)^{\nu-1}=-\frac{3(1+w) Z_{*}}{\left(1-Z_{*}^{2}\right)^{\frac{3}{2}-\nu}} \\
(1+3 w) \omega_{m *}^{2}+(1-3 w)\left[1-\frac{6 \beta^{2}}{\mu}\left(1-Z_{*}^{2}\right)\right] \omega_{m *}-2\left[1-\left(1-Z_{*}^{2}\right)^{2}\right]
\end{gathered}
$$

They exist only for $A Z_{*}<0$ and correspond to the ones given by equations (42)-(44). For the physically interesting case $w=0$ with influx we scanned the parameter space and were convinced that for $\nu \neq 3 / 2$ there is always only one fixed point; for $\nu<3 / 2$ this is an attractor (A), while for $\nu>3 / 2$ this is a saddle (S). For $w=0, \nu=3 / 2$ there is either one fixed point (attractor) or no fixed points, depending on the parameters. For the other characteristic value $w=1 / 3$, we concluded that for $\nu<3 / 2$ there is only one fixed point (attractor), for $\nu>2$ there is only one fixed point (saddle), while for $3 / 2<\nu<2$ there are either two fixed points (one attractor and one saddle) or no fixed points at all, depending on the parameters. For $w=1 / 3, \nu=3 / 2$ there is either one fixed point (attractor) or no fixed points. Finally, for $w=1 / 3, \nu=2$ there is either one fixed point (saddle) or no fixed points. These results were obtained numerically for a wide range of parameters and are summarized in Tables 1 and 2 .

\begin{tabular}{|c|c|c|c|}
\hline & $\nu<3 / 2$ & $\nu=3 / 2$ & $\nu>3 / 2$ \\
\hline No. of F.P. & 1 & 0 or 1 & 1 \\
\hline Nature & $\mathrm{A}$ & $\mathrm{A}$ & $\mathrm{S}$ \\
\hline
\end{tabular}

Table 1: The fixed points for $\mathrm{w}=0$, influx

\begin{tabular}{|c|c|c|c|c|c|}
\hline & $\nu<3 / 2$ & $\nu=3 / 2$ & $3 / 2<\nu<2$ & $\nu=2$ & $\nu>2$ \\
\hline No. of F.P. & 1 & 0 or 1 & 0 or 2 & 0 or 1 & 1 \\
\hline Nature & $\mathrm{A}$ & $\mathrm{A}$ & $\mathrm{A}, \mathrm{S}$ & $\mathrm{S}$ & $\mathrm{S}$ \\
\hline
\end{tabular}

Table 2: The fixed points for $\mathrm{w}=1 / 3$, influx

The approach to an attractor described by the linear approximation of (51)-(52) is exponential in $\tau$ and takes infinite time $\tau$ for the universe to reach it. Given that near this fixed point the relation between the cosmic time $t$ and the time $\tau$ is linear, we conclude that it also takes infinite cosmic time to reach the attractor.

Defining $\epsilon=\operatorname{sgn}(H)$, we see from (51)-(52) that the lines $Z=\epsilon(\nu \leq 3 / 2), \omega_{m}=0$ are orbits of the system. Furthermore, the family of solutions with $Z \approx \epsilon$ and $d Z / d \omega_{m}=Z^{\prime} / \omega_{m}^{\prime} \approx 0$ is approximately described for $\nu<3 / 2$ by $\omega_{m}^{\prime}=\epsilon(1+3 w) \omega_{m}\left(\omega_{m}-1\right)$, and thus, they move away from the point $\left(\omega_{m *}, Z_{*}\right)=(1,1)$, while they approach the point $\left(\omega_{m *}, Z_{*}\right)=(1,-1)$. In addition, the solution of this equation is $\omega_{m}=\left[1+c e^{\epsilon(1+3 w) \tau}\right]^{-1}$, with $c>0$ an integration constant. Using this solution in equation $H^{\prime} / H=-Z(q+1)$ we find that for $w=1 / 3$, $H / H_{o}=\sqrt{\omega_{m}} /\left(1-\omega_{m}\right)$, where $H_{o}$ is another integration constant. Then, the equation for $\omega_{m}(t)$ becomes $d \omega_{m} / d t=-2 \epsilon \omega_{m} \sqrt{H_{o}^{2} \omega_{m}-\mu\left(1-\omega_{m}\right)^{2}}$, and can be integrated giving $t$ as a function of $\omega_{m}$ or $H$. Therefore, in the region of the big bang/big crunch singularity one obtains $a(t) \sim \sqrt{\epsilon t}, \rho(t) \sim t^{-2}$, as in the standard radiation dominated big-bang scenario. This means that for $\nu<3 / 2$ the energy exchange has no observable effects close to the big bang/big crunch singularity.



FIG. 1: Influx, $w=0, \nu<3 / 2$. The arrows show the direction of increasing cosmic time. The dotted line corresponds to $w_{D E}=-1$. The region inside (outside) the dashed line corresponds to acceleration (deceleration). The region with $Z>0$ represents expansion, while $Z<0$ represents collapse. The present universe is supposed to be close to the global attractor.

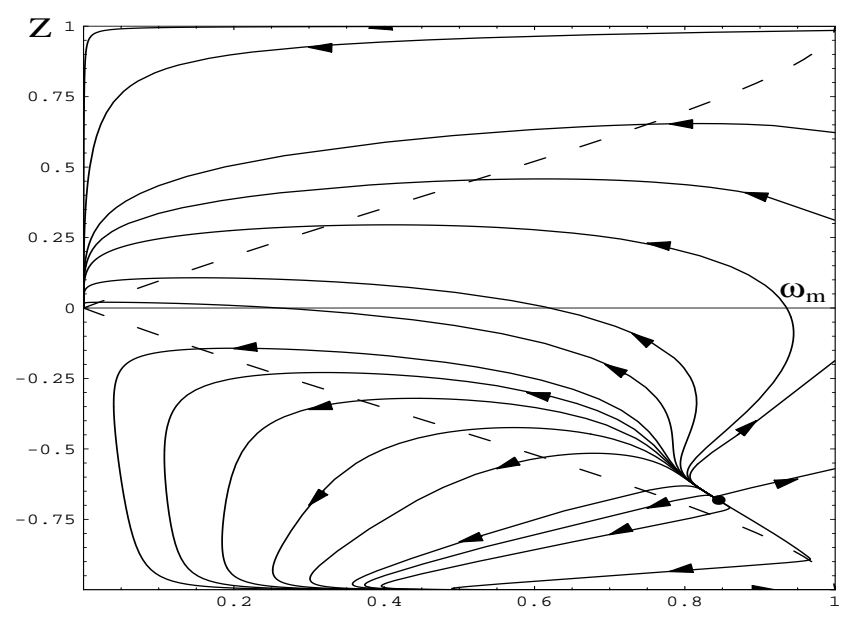

FIG. 2: Outflow, $w=1 / 3, \nu<3 / 2$. The arrows show the direction of increasing cosmic time. The region inside (outside) the dashed line corresponds to acceleration (deceleration). The region with $Z>0$ represents expansion, while $Z<0$ represents collapse.

Since our proposal relies on the existence of an attractor, we shall restrict ourselves to the case $\nu<3 / 2$. It is convenient to discuss the four possible cases separately: (i) $w=0$ with influx. The generic behavior of the solutions of equations (51)-(52) is shown in Figure 1. We 
see that all the expanding solutions approach the global attractor. Furthermore, there is a class of collapsing solutions which bounce to expanding ones. Finally, there are solutions which collapse all during their lifetime to a state with finite $\rho$ and $H$. The physically interesting solutions are those in the upper part of the diagram emanating from the big bang $(\omega, Z) \approx(1,1)$. These solutions start with a period of deceleration. The subsequent evolution depends on the value of $3 \beta^{2} /|\mu|$, which determines the relative position of the dashed and dotted lines. Specifically, for $3 \beta^{2} /|\mu|>1$ (the case of Figure 1) one distinguishes two possible classes of universe evolution. In the first, the universe crosses the dashed line entering the acceleration era still with $w_{D E}>-1$, and finally it crosses the dotted line to $w_{D E}<-1$ approaching the attractor. In the second, while in the deceleration era, it first crosses the dotted line to $w_{D E}<-1$, and then the dashed line entering the eternally accelerating era. For $3 \beta^{2} /|\mu| \leq 1$, the dotted line lies above the dashed line, and, consequently, only the second class of trajectories exists. To connect with the discussion in the introduction, notice that the Friedmann equation (39) can be written in the form (11) with dark energy $\rho_{D E}=(\beta \psi+\mu) / 2 \gamma$. Using (40), the equation for $\rho_{D E}$ takes the form (3) with

$w_{D E}=\frac{-1}{3\left(1-\omega_{m}\right)}\left[2 Z^{2}-\omega_{m}-1-6(1-3 w) \frac{\beta^{2}}{\mu} \frac{\omega_{m}\left(1-Z^{2}\right)}{Z^{2}-\omega_{m}}\right]$.

The global attractor (42)-(44) satisfies relations (44) and consequently, $w_{D E}$ evolves to the value $w_{D E *}$ given by (5). As for the bouncing solutions, they approach the attractor after they cross the line $Z^{2}=\omega_{m}$, where $w_{D E}$ jumps from $+\infty$ to $-\infty$; however, the evolution of the observable quantities is regular.

(ii) $w=0$ with outflow. The generic behavior in this case is obtained from Figure 1 by the substitution $Z \rightarrow-Z$ and $\tau \rightarrow-\tau$, which reflects the diagram with respect to the $\omega_{m}$ axis and converts attractors to repelers.

(iii) $w=1 / 3$ with outflow. Figure 2 depicts the flow diagram of this case. Even though in the case of radiation in general $w_{D E}>-1 / 3$ from equation (56), there are both acceleration and deceleration regions. Furthermore, from equation (5) it is $\Omega_{m *}>1$.

(iv) $w=1 / 3$ with influx. This arises like in (ii) by reflection of Figure 2 and resembles Figure 1.

\section{CONCLUSIONS}

In this work, we studied the role of brane-bulk energy exchange on the cosmological evolution of a brane with negative tension, zero effective cosmological constant, and in the presence of the induced curvature scalar term in the action. Adopting the physically motivated $\rho^{\nu}$ power-law form for the energy transfer and assuming a cosmological constant in the bulk, an autonomous system of equations was isolated. In this scenario, the "dark energy" is a result of the geometry and the brane-bulk energy exchange. The negative tension of the brane is necessary in order to realize the present universe (accelerating with $0<\Omega_{m 0}<1$ ) as being close to a future fixed point of the evolution equations. We studied the possible cosmologies using bounded normalized variables and the corresponding global phase portraits were obtained. By studying the number and nature of the fixed points we demonstrated numericaly that our present universe can be easily realized as a late-time fixed point of the evolution. This provides an alternative answer to the coincidence problem in cosmology, which does not require specific fine-tuning of the initial data. Furthermore, the equation of state for the dark energy at the attractor is uniquely specified by the value $\Omega_{m 0}$. Remarkably, for $\Omega_{m 0}=0.3$, one obtains $w_{D E, 0}=-1.4$, independently of the other parameters, while for the other suggestive value $\Omega_{m 0}=0.03, w_{D E, 0}=-1.03$. In the past, the function $w_{D E}$ crosses the line $w_{D E}=-1$ to larger values.

It would be interesting to investigate if the above partial success of the present scenario persists after one tries to fit the supernova data and the detailed CMB spectum 22]. Of course, the nature of the content of the bulk and of the mechanism of energy exchange with the brane is another crucial open question, which we hope to deal with in a future publication.

Acknowlegements. Supported in part by the EU grant MRTN-CT-2004-512194. The work of G.K. is also supported by the European Commission Marie-Curie Fellowship under contract MEIF-CT-2004-501432. G.P. is also supported by the Greek Ministry of Education research program "Herakleitos".
[1] J. Ellis, S. Kalara, K. Olive and C. Wetterich, Phys. Lett. B228 (1989) 264; C. Wetterich, Astron. Astrophys. 301 (1995) 321 hep-th/9408025; L. Amendola, Phys. Rev. D60 (1999) 043501 astro-ph/9904120; L. Amen- dola and D. Tocchini-Valentini, Phys. Rev. D64 (2001) 043509 astro-ph/0011243; L. Chimento, A. Jakubi, D. Pavon and W. Zimdahl, Phys. Rev. D67 (2003) 083513 astro-ph/0303145; L. Amendola, M. Gasperini and F. 
Piazza, astro-ph/0407573

[2] E. Kiritsis, G. Kofinas, N. Tetradis, T.N. Tomaras and V. Zarikas, JHEP 0302 (2003) 035 hep-th/0207060.

[3] This is in contrast to the models discused in [1] in which equation (4) is not satisfied.

[4] U. Alam, V. Sahni and A. Starobinsky, JCAP 0406 (2004) 008 astro-ph/0403687; Y. Gong, Class. Quant. Grav. 22 (2005) 2121 astro-ph/0405446; Y. Wang and M. Tegmark, Phys. Rev. D71 (2005) 103513 astro-ph/0501351; Y. Wang and P. Mukherjee, Astrophys. J. 606 (2004) 654 astro-ph/0312192; R. Daly and S. Djorgovski, Astrophys. J. 612 (2004) 652 astro-ph/0403664; U. Alam, V. Sahni, T. Saini and A. Starobinsky, Mon. Not. Roy. Astron. Soc. 354 (2004) 275 astro-ph/0311364; T. Choudhury and T. Padmanabhan, Astron. Astrophys. 429 (2005) 807 astro-ph/0311622.

[5] A. Riess et all, Astrophys. J. 607 (2004) 665 astro-ph/0402512; S. Allen, R. Schmidt, H. Ebeling, A. Fabian and L. van Speybroeck, Mon. Not. Roy. Astron. Soc. 353 (2004) 457 astro-ph/0405340; A. Melchiorri, L. Mersini, C. Odman and M. Trodden, Phys. Rev. D68 (2003) 043509 astro-ph/0211522; P. Singh, M. Sami and N. Dadhich, Phys. Rev. D68 (2003) 023522 hep-th/0305110; E. Gunzig and A. Saa, Int. J. Mod. Phys. D13 (2004) 2255 gr-qc/0406068; F. Carvalho and A. Saa, Phys. Rev. D70 (2004) 087302 astro-ph/0408013.

[6] S. Carroll, M. Hoffman and M. Trodden, Phys. Rev. D68 (2003) 023509 astro-ph/0301273; D. Huterer and A. Cooray, Phys. Rev. D71 (2005) 023506 astro-ph/0404062; H. Jassal, J. Bagla and T. Padmanabhan, Mon. Not. Roy. Astron. Soc. 356 (2005) L11 astro-ph/0404378; Y. Gong, Int. J. Mod. Phys. D14 (2005) 599; P. Corasaniti, M. Kunz, D. Parkinson, E. Copeland and B. Bassett, Phys. Rev. D70 (2004) 083006 astro-ph/0406608; S. Hannestad and E. Mortsell, JCAP 0409 (2004) 001 astro-ph/0407259; Y. Gong and Y. Zhang, astro-ph/0502262

[7] R. Lazkoz, S. Nesseris and L. Perivolaropoulos, astro-ph/0503230 L. Perivolaropoulos, astro-ph/0504582

[8] C. Armendariz-Picon, T. Damour and V. Mukhanov, Phys. Lett. B458 (1999) 209 hep-th/9904075; J. Garriga and V. Mukhanov, Phys. Lett. B458 (1999) 219 hep-th/9904176; L. Parker and A. Raval, Phys. Rev. D60 (1999) 063512 [Erratum-ibid. D67 (2003) 029901] gr-qc/9905031; R. Caldwell, Phys. Lett. B545 (2000) 23 astro-ph/9908168; C. Armendariz-Picon, V. Mukhanov and P. Steinhardt, Phys. Rev. Lett. 85 (2000) 4438 astro-ph/0004134; A. Schulz, M. White, Phys. Rev. D64 (2001) 043514 astro-ph/0104112; I. Maor, R. Brustein, J. McMahon and P. Steinhardt, Phys. Rev. D65 (2002) 123003 astro-ph/0112526; V. Sahni and Y. Shtanov, JCAP 0311 (2003) 014 astro-ph/0202346; P. Frampton, Phys. Lett. B555 (2003) 139 astro-ph/0209037; M. Ahmed, S. Dodelson, P. Greene and R. Sorkin, Phys. Rev. D69 (2004) 103523 astro-ph/0209274; B. Feng, X-L Wang and X-M Zhang, Phys. Lett. B607 (2005) 35 astro-ph/0404224; V. Onemli and R. Woodard, Phys. Rev. D70 (2004) 107301 gr-qc/0406098; A. Vikman, Phys. Rev. D71 (2005) 023515 astro-ph/0407107; S. Nojiri and S. Odintsov, Phys. Rev. D70 (2004)
103522 hep-th/0408170; A. Lue and G. Starkman, Phys. Rev. D70 (2004) 101501 astro-ph/0408246; Z. Guo, Y. Piao, X. Zhang and Y. Zhang, Phys. Lett. B608 (2005) 177 astro-ph/0410654; W. Hu, Phys. Rev. D71 (2005) 047301 astro-ph/0410680; S. Nojiri, S. Odintsov and S. Tsujikawa, Phys. Rev. D71 (2005) 063004 hep-th/0501025; H. Wei, R-G Cai and D-F Zeng, Class. Quant. Grav. 22 (2005) 3189 hep-th/0501160; X. Zhang, H. Li, Y. Piao and X. Zhang, astro-ph/0501652 P. Singh, gr-qc/0502086 M. Li, B. Feng and X. Zhang, hep-ph/0503268 I. Brevik and O. Gorbunova, gr-qc/0504001 H. Stefancic, Phys. Rev. D71 (2005) 124036 astro-ph/0504518; A. Anisimov, E. Babichev and A. Vikman, JCAP 0506 (2005) 006 astro-ph/0504560; R. Cai, H. Zhang and A. Wang, hep-th/0505186 B. Wang, Y. Gong and E. Abdalla, hep-th/0506069 M. Cataldo, N. Cruz and S. Lepe, Phys. Lett. B619 (2005) 5 hep-th/0506153.

[9] G. Dvali, G. Gabadadze and M. Porrati, Phys. Lett. B485 (2000) 208 hep-th/0005016; G. Dvali and G. Gabadadze, Phys. Rev. D63 (2001) 065007 hep-th/0008054.

[10] L. Hall and D. Smith, Phys. Rev. D60 (1999) 085008 hep-ph/9904267; S. Hannestad, Phys. Rev. D64 (2001) 023515 hep-ph/0102290.

[11] U. Ellwanger, Eur. Phys. J. C25 (2002) 157 hep-th/0001126; C. Barcelo and M. Visser, Phys. Rev. D63 (2001) 024004 gr-qc/0008008; K. Maeda and D. Wands, Phys. Rev. D62 (2000) 124009 hep-th/0008188; A. Mennin and R. Battye, Class. Quant. Grav. 18 (2001) 2171 hep-th/0008192; C. van de Bruck, M. Dorca, C. Martins and M. Parry, Phys. Lett. B495 (2000) 183 hep-th/0009056; A. Hebecker and J. March-Russel, Nucl. Phys. B608 (2001) 375 hep-ph/0103214; P. Brax, C. van de Bruck and A. C. Davis, JHEP 0110 (2001) 026 hep-th/0108215; D. Langlois, L. Sorbo and M. Rodriguez-Martinez, Phys. Rev. Lett. 89 (2002) 171301 hep-th/0206146; E. Leeper, R. Maartens and C. Sopuerta, Class. Quant. Grav. 21 (2004) 1125 gr-qc/0309080; P. Binetruy, M. Bucher and C. Carvalho, Phys. Rev. D70 (2004) 043509 hep-th/0403154; M. Bouhmadi-Lopez and D. Wands, Phys. Rev. D71 (2005) 024010 hep-th/0408061.

[12] P. Apostolopoulos and N. Tetradis, Class. Quant. Grav. 21 (2004) 4781 hep-th/0404105; F. Diakonos, E. Saridakis and N. Tetradis, Phys. Lett. B605 (2005) 1 hep-th/0409025; E. Kiritsis, hep-th/0504219

[13] E. Kiritsis, N. Tetradis and T.N. Tomaras, JHEP 0203 (2002) 019 hep-th/0202037.

[14] C. Deffayet, Phys. Lett. B502 (2001) 199 hep-th/0010186; C. Deffayet, G. Dvali and G. Gabadadze, Phys. Rev. D65 (2002) 044023 astro-ph/0105068.

[15] V. Sahni and Y. Shtanov, Int. J. Mod. Phys. D11 (2000) 1515 gr-qc/0205111.

[16] L. Pilo, R. Rattazzi and A. Zaffaroni, JHEP 0007 (2000) 056 hep-th/0004028; Y. Shtanov and A. Viznyuk, Class. Quant. Grav. 22 (2005) 987 hep-th/0312261; A. Padilla, Class. Quant. Grav. 21 (2004) 2899 hep-th/0402079.

[17] C. Charmousis and J-F Dufaux, Phys. Rev. D70 (2004) 106002 hep-th/0311267.

[18] J. Lehners, P. Smyth and K. Stelle, Class. Quant. Grav. 22 (2005) 2589 hep-th/0501212.

[19] M. Smolyakov and I. Volobuev, hep-th/0208025 E. Kir- 
itsis, N. Tetradis and T.N. Tomaras, JHEP 0108 (2001) 012 hep-th/0106050; G. Kofinas, E. Papantonopoulos and I. Pappa, Phys. Rev. D66 (2002) 104014 hep-th/0112019.

[20] M. Goliath and G.F.R. Ellis, Phys. Rev. D60 (1999) 023502 gr-qc/9811068; A. Campos and C. Sopuerta, Phys. Rev. D63 (2001) 104012 hep-th/0101060; N. Go- heer and P. Dunsby, Phys. Rev. D66 (2002) 043527 gr-qc/0204059.

[21] L. Perko, "Differential equations and dynamical systems", Springer-Verlag, 1991.

[22] K. Umezu, K. Ichiki, T. Kajino, G. Mathews, R. Nakamura and M. Yahiro, astro-ph/0507227 